\title{
Estrategias de mercadeo implementadas por las empresas de servicios de la Gran Área Metropolitana en Costa Rica
}

\section{Marketing strategies implemented by service companies in the Greater Metropolitan Area of Costa Rica}

Dra. Ana Hernández Mainieri

Universidad Fidélitas, Costa Rica

hernandezmainieri@gmail.com
Mag. Geiner Mora Miranda

Universidad Fidélitas, Costa Rica

geinermmo8@gmail.com

\section{RESUMEN:}

El mercadeo, al igual que muchas áreas del conocimiento humano, ha venido evolucionando a lo largo de los años en la búsqueda de mejores estrategias que contribuyan eficientemente con el logro de los objetivos organizacionales. La gestión mercadológica dentro del quehacer empresarial, exige mayor conocimiento por parte de los administradores, de manera que se posibilite la implementación de estrategias más eficientes.

En este caso, se analiza el modo en que los administradores de empresas servicios de algunas empresas de diversos sectores de la Gran Área Metropolitana costarricense dicen emplear los elementos de las 4 C en sus organizaciones Por lo anterior, se realiza la revisión cualitativa de las estrategias mercadológicas que emplean quince empresas de servicios de la Gran Área Metropolitana, a la luz de la estrategia recomendado por Cobra (2000), conocida como $4 \mathrm{C}$, y, con base en ello, se realiza una propuesta para ser empleada por las empresas costarricenses en forma funcional.

\section{ABSTRACT:}

Marketing, like many areas of human knowledge, has evolved along the years in search of better strategies that may contribute efficiently to reach organizational goals. Marketing management within the work of business demands greater knowledge from administrators so as to enable the implementation of more efficient strategies.

In this case, we analyze the way in which managers of some service companies of various sectors of the Greater Metropolitan Area of Costa Rica claim to use the $4 \mathrm{C}$ elements in their organizations. Therefore, the qualitative revision of the marketing strategies used by fifteen service companies of the Greater Metropolitan Area are reviewed in the light of the strategy recommended by Cobra (2000) and known as $4 \mathrm{C}$. Based on this, a proposal is made to be used by Costa Rican companies in a functional manner.

\section{RESUMO:}

O marketing, como muitas áreas do conhecimento humano, evoluiu ao longo dos anos na busca de melhores estratégias que contribuam eficientemente com o sucesso dos objetivos organizacionais. A gestão mercadológica nas tarefas empresariais, requer maior conhecimento dos administradores, para permitir que se possibilite a implementação de estratégias mais eficientes.

Nesse caso, é analisada a maneira pela qual os administradores de serviços de algumas empresas de vários setores da Grande Área Metropolitana costarriquenha dizem que usam os elementos dos 4 C em suas organizações.

Devido a isto, realiza-se uma revisão qualitativa das estratégias mercadológicas que empregam quinze empresas de serviços da Grande Área Metropolitana, à luz da estratégia recomendada por Cobra (2000), conhecida como $4 \mathrm{C}$, e, baseado nisto, é feita uma proposta para ser usada pelas empresas costarriquenhas de maneira funcional

\section{RÉSUMÉ:}

La commercialisation, à l'instar de nombreux domaines de la connaissance humaine, a évolué au long des années dans la recherche de meilleures stratégies qui puissent contribuer efficacement à atteindre les objectifs organisationnels. La gestion commerciale au sein des activités de l'entreprise, exige une plus grande connaissance de la part des administrateurs, afin de pouvoir mettre en ouvre des stratégies plus efficaces.

Dans ce cas, on analyse la façon selon laquelle les administrateurs de certaines entreprises de service dans différents secteurs de la grande région métropolitaine costaricienne affirment utiliser les éléments des 4 C dans leurs organisations. C'est pourquoi, on effectue la révision qualitative des stratégies commerciales que quinze sociétés de service de la grande région métropolitaine utilisent à la lumière de la stratégie recommandée par Cobra (2000) et connue comme $4 \mathrm{C}$. Sur cette base, on effectue une proposition devant être utilisée par les sociétés costariciennes de manière fonctionnelle.

\section{PALABRAS CLAVES}

ESTRATEGIA DE 4 P, ESTRATEGIA DE 4 C, COSTOS BASADOS EN ACTIVIDADES; ADMINISTRACIÓN BASADA EN ACTIVIDADES CADENA DE VALOR

\section{KEYWORDS}

4 P STRATEGY, 4 C STRATEGY, ACTIVITY

BASED COSTS, ACTIVITY BASED ADMINISTRATION VALUE CHAIN

\section{PALAVRAS CHAVES}

ESTRATÉGIA DE 4 P, ESTRATÉGIA DE 4 C, CUSTOS BASEADOS EM ATIVIDADES; ADMINISTRAÇÃO BASEADA EM ATIVIDADES CADEIA DE VALOR

\section{MOTS CLÉS}

STRATEGIE 4 P, STRATEGIE $4 \mathrm{C}$, COUTS BASES SUR LES ACTIVITES, ADMINISTRATION BASEE SUR LES ACTIVITES, CHAINE DE VALEUR 


\section{INTRODUCCIÓN}

Gracias a los avances científicos y tecnológicos que se han generado a partir de la segunda mitad del siglo XX, se ha dado un cambio en la estructura de producción de las organizaciones. En el pasado era frecuente observar que las empresas exitosas desarrollaran productos (tangibles) para ofertar al mercado; sin embargo, en la actualidad, el crecimiento del sector servicios (intangibles) es evidente (Beverinotti, Chang, Corrales y Vargas, 2014).

Hoy, las empresas suelen apostar por los servicios como una forma de alcanzar ventaja competitiva y así permanecer dentro de los mercados, situación que hace necesario realizar un recorrido por los mecanismos que emplean las empresas de servicios, la naturaleza de los estos y el abordaje teórico que permita proponer una estrategia para ser empleado por las empresas costarricenses en forma funcional.

Generalmente, las organizaciones que ofrecen servicios tienden a administrar su estrategia de comunicación integrada de mercadeo (CIM) como si fuesen tangibles, es decir, con las tradicionales cuatro P (Kotler y McCarthy, 2016. Sin embargo, autores como Cobra (2000), Lovelock (2016) y Hoffman (2002), manifiestan que los servicios requieren un tratamiento mercadológico particular.

Lo que se pretende, entonces, con este estudio, es realizar un diagnóstico de la forma en que los administradores de las empresas costarricenses están gestionando el mercadeo de servicios y, a partir de ahí, identificar una estrategia que facilite la comunicación funcional con los públicos atendidos, de manera que se logren alcanzar los objetivos estratégicos formulados en el proceso de planificación.

Debido a la naturaleza y características de los servicios, su estrategia de mercadotecnia requiere un tratamiento diferente al que usualmente se emplea en los productos. Los servicios son intangibles que requieren ser percibidos por los clientes, por lo que generalmente se hace uso de elementos diferenciadores tales como infraestructura y equipamiento, tecnología y recurso humano (Hoffman, 2002).

Entre las características principales de los servicios, se puede mencionar que son intangibles, heterogéneos, imperdurables, se evalúan hasta que se consumen, son inseparables, difíciles de estandarizar y de establecer su precio. Es decir, la estrategia de mercadeo que se implemente en estos debe ser adaptada a su naturaleza y diferenciada de las utilizadas para promocionar productos.

Cobra (2000), sostiene que la comunicación que se desarrolla al promocionar tangibles debe ser adaptada en el caso de los servicios, empleando las 4 C (cliente, costos, comodidad y comunicación). Para ello, se requiere la identificación precisa del mercado objetivo, de manera tal que se conozcan los requerimientos particulares de los clientes, ya sea que pertenezcan al mercado organizacional (industria, revendedores, Gobierno/instituciones e internacional) o al de consumidores (Kotler, 2017).

\section{SUSTENTO TEÓRICO}

De acuerdo con Kotler, Bloom y Hayes (2016), "un servicio es una obra, una realización o un acto que es esencialmente intangible y no resulta necesariamente en la propiedad de algo. Su creación puede o no estar relacionada con un producto físico" (pág. 9). Los servicios son intangibles, por lo que se requiere del apoyo de "tangibilizadores" que faciliten ser percibidos por parte de los clientes.

A partir de la Segunda Guerra Mundial, con la revolución tecnológica (Tofler, 1997) se ha dado el desarrollo de los servicios como elemento esencial en el quehacer de las organizaciones, esto gracias a la sustitución de la economía del trabajo por la economía del conocimiento en las sociedades avanzadas. Por ello, es necesario mencionar las características de los servicios, de manera que el mercadólogo ejecute estrategias adecuadas a los públicos servidos. 


\section{Características de los servicios}

Autores como Lovelock (2016) señalan que, entre las características de los servicios se pueden mencionar:

Intangibilidad: difícilmente se perciben por medio de los sentidos.

Perecederos: se consumen en el momento en que se prestan.

Heterogeneidad: es difícil su estandarización debido a que generalmente son ofrecidos y recibidos por personas.

Inseparabilidad: casi siempre son ofrecidos por personas, por lo que se dificulta separar el servicio de las características personales de quien lo ofrece.

Dificultad de estandarización: al ser ofrecido y recibido por personas, hay diferencias sustanciales en la personalidad, conocimiento y características generales de ambas partes. Asimismo, un solo proveedor puede tener rendimientos diferenciados en función del nivel de "cansancio" que posee; por ejemplo, el servicio que ofrece por una persona en horas de la mañana, no necesariamente va a ser igual al que da por la noche.

Dificultad de establecer su precio: al ser difícil la estandarización, se dificulta automáticamente el establecimiento del precio, principalmente en cuanto a la variable de costos, como una de las determinantes en su fijación.

Evaluación después del consumo: en el caso de los productos- tangibles, es posible percibir antes de la compra (tocar, oler, medir, palpar y otros) el nivel de satisfacción y valor que generará ese bien. En tanto que en los servicios, al ser intangibles, heterogéneos e inseparables, la satisfacción percibida por el comprador se genera hasta que se consume.

Considerando estas características, las empresas deben garantizarse que el mercado objetivo comprende los elementos diferenciadores del servicio.

\section{Estrategias de diferenciación en servicios}

Hoffman y Bateson (2012) señalan que, entre las estrategias de diferenciación que pueden emplear las organizaciones de servicios, se encuentran las siguientes:

Recurso humano: contratar a los mejores candidatos de acuerdo con las características particulares de los servicios y del segmento objetivo. Personas con capacidad de toma de decisiones, por supuesto dentro del ámbito de acción de su puesto de trabajo; empoderadas, inteligentes emocionalmente, hábiles a nivel de empatía, escucha y observación, entre otros.

Infraestructura y equipamiento: los edificios, oficinas, parqueos, vehículos, equipos, colores, diseños y acceso a edificaciones, deben poseer las condiciones necesarias para personas con alguna discapacidad física, entre otros, y deben estar acordes con lo que la empresa desea comunicar al mercado.

Tecnología: los instrumentos, recursos técnicos y procedimientos empleados por la empresa de servicios deben estar alineados con las características del mercado objetivo y los objetivos de la empresa.

Considerando estos criterios, es posible entonces que las organizaciones logren sus objetivos estratégicos y permanezcan en el mercado; por supuesto, siempre teniendo como eje central la gestión dirigida a los clientes, con sus necesidades y deseos, de forma que el servicio se ajuste a ellos. Es decir, creando un balance entre los objetivos de la empresa y las necesidades particulares de los clientes, bajo una visión ganar-ganar.

\section{Estrategia de $4 \mathrm{C}$}

A nivel mercadológico, se menciona que las herramientas fundamentales de trabajo están asociadas con la mezcla de mercadeo, también conocida como las $4 \mathrm{P}$ (producto, precio, plaza y promoción). Sin embargo, al comercializar servicios, las empresas deben ser conscientes de la particularidad de estos en cuanto a sus características distintas a los 
tangibles, las cuales ya se mencionaron con anterioridad. Por ello, Cobra (2000) señala que, para poder ser competitivo en una empresa dedicada a ofrecer servicios, se recomienda trabajar con $4 \mathrm{C}$ en lugar de $4 \mathrm{P}$.

\section{Las 4 C: cliente, costos, comodidad y comunicación}

Cuando se habla de clientes, se hace referencia a las personas físicas o jurídicas que conforman el mercado objetivo de la organización y a las cuales se ofertan los servicios. Vale la pena recordar que se suelen atender a dos grandes tipos de mercados: consumidores, que están conformados por muchas personas físicas (hogares) que adquieren pocas cantidades de producto, y el mercado organizacional o de negocios, conformado por personas físicas o jurídicas que adquieren grandes volúmenes de servicios. Así, es fundamental tipificar las características del segmento de mercado al cual se pretende servir, de forma tal que el cliente logre percibir su diferenciación.

La $\mathrm{C}$ de costos contribuye con la fijación y el ajuste de precios. Asimismo, es posible considerar el precio psicológico, el nivel del mercado y la demanda, y los objetivos de la alta dirección. En otras palabras, el precio puede variar en función de quién lo proporcione y quién los reciba. Se deben considerar al menos los costos fijos, los variables y los totales.

Los costos se clasifican en costos fijos, costos variables y costos mixtos. Los costos fijos son aquellos que permanecen constantes durante cierto período, independientemente de los cambios presentados en el volumen de producción; tal es el caso de los costos por concepto de arrendamiento y primas de seguros, entre otros. Los costos variables totales son los que oscilan durante cierto período en función de los cambios presentados en el nivel de actividad; por ejemplo, los costos de materia prima, de combustible y otros.

En cuanto a la comodidad, se debe ofrecer un servicio al cliente de excelencia, caracterizado por el personal eficiente, capacitado, cortés, amable, de manera que se cumpla con las expectativas del consumidor y, por lo tanto, se genere satisfacción y valor. Además, es posible establecer varios puntos de venta cercanos a los clientes, servicios personalizados o "hechos a la medida", marketing relacional y radical (Hill, 2000). Para hacer más cómodo el servicio, se desarrollan también modelos de merchandising.

Entiéndase por merchandising el "producto/servicio en movimiento", es decir, colocado en el punto de venta de una forma tan atractiva que se venda solo. Así, es posible facilitar el acceso de los clientes a los servicios, por medio de estímulos sensoriales que afecten oído, olfato, visión, tacto y gusto, en función del perfil de consumidores a los cuales está dirigida la oferta.

La comunicación, debe ser definida en forma estratégica, considerando los medios idóneos de acuerdo con el mercado meta, definiendo las estrategias y tácticas de publicidad, relaciones públicas, promoción de ventas, venta personal y mercadeo directo.

\section{Estrategia de comunicación integrada de mercadeo}

Está conformada por la mezcla promocional, que, en el caso de servicios, se asocia directamente con procesos de comunicación funcional e incluye:

Publicidad: referida la difusión o divulgación de información de cualquier naturaleza, en forma unidireccional, empleando medios masivos pagados, con un patrocinador identificado. Entre esos medios masivos, es posible mencionar televisión, prensa (periódicos y revistas), radio, Internet y medios exteriores (Treviño, 2010).

Relaciones públicas: enfocada en la promoción de la imagen pública de las organizaciones, por medio del trato personalizado. Como afirma Wilcox (2012), se refiere a la gestión de la comunicación entre la empresa y sus públicos.

Promoción de ventas: De acuerdo con Rivera Camino y de Juan Vigaray (2002) es "el conjunto de técnicas que refuerzan y animan la oferta normal de la empresa, con el objetivo de incrementar la venta [...] en el corto plazo” (pág. 17) Entre esas técnicas, es posible mencionar las siguientes: muestras gratis, pruebas, concursos, regalos y precios especiales. 
Venta personal: se caracteriza porque un vendedor ofrece, promociona y vende en forma directa (cara a cara) un servicio a un consumidor u organización. Este proceso de venta incluye una serie de fases que van desde la prospección, el preacercamiento, la presentación de ventas (que puede ser individual o por sistema), el manejo de objeciones, el cierre y el seguimiento o servicios posventa.

La competencia actualmente es muy fuerte, por lo que es necesario lograr diferenciarse por medio de la oferta de los satisfactores deseados por el mercado objetivo. Los clientes están cada vez más informados y con mayores exigencias, pues fácilmente pueden acceder a fuentes que les nutren de los datos requeridos para efectuar una compra. La competencia y la tecnología han evolucionado; así que las empresas no deben quedarse solo con haber podido vender un servicio, sino generar compras repetitivas por parte del mismo cliente y conservarlo. "A tus clientes no les gusta que les des un mal servicio, pero a tu competencia sî”., (Zabriskie, 2009, citado por Córdoba López, 2009).

\section{Importancia de los costos}

Según Govindarajan y Shank (1998), la gerencia estratégica de costos consiste en el uso que la alta dirección de una organización hace de la información de costos, para tomar decisiones que incrementen el valor de los accionistas, y resulta de la combinación de tres temas: análisis de la cadena de valor, análisis de posicionamiento estratégico y análisis de causales de costos.

La cadena de valor es un método para la definición de las actividades que se desarrollan en la empresa de forma estratégica, con el objeto de comprender el comportamiento de los costos y las fuentes de diferenciación. Así, se hace posible tomar decisiones enfocadas en mantener ventajas competitivas sostenibles, por lo que se constituye en el eje de la gerencia estratégica de costos.

Morillo (2005) señala que, con la tecnología, la administración basada en actividades (ABC) toma un carácter complejo en el proceso de identificar y ubicar las actividades de la empresa y se integra con el sistema de administración basada en actividades (ABM), permitiendo la compatibilidad estrecha con la estructura de la cadena de valor.

El primer objetivo de la gestión estratégica de costos es organizar la información para que la empresa mantenga la competitividad a través de la mejora continua de productos y servicios de alta calidad que satisfagan a los clientes al menor precio. Este objetivo busca y mantiene la excelencia empresarial, la cual se logra mediante el diseño y la adaptación continua de una combinación productiva global que mantenga el valor percibido por el cliente y que le permita anticiparse a los cambios.

La dinámica para la determinación de los costos en las empresas de servicios es más compleja y diversa que en el caso de productos/tangibles, dado el tipo de actividad y la diversidad de los servicios; por lo que se hace necesario considerar características propias de cada empresa. Dentro de los principales elementos de costos que se pueden encontrar en las empresas de servicios, se tienen los costos directos, los indirectos y las operaciones. El marketing de los servicios exige entonces imaginación y habilidad administrativa a la hora de definir su precio. Características como caducidad, almacenamiento y demanda hacen que se dificulte aún más. 


\section{METODOLOGÍA}

El interés en desarrollar este artículo es el establecer la estrategia de administración de mercadeo y costos de servicios para ser implementado por las empresas de servicios de la GAM.

Los objetivos específicos son los siguientes:

1. Identificar el perfil de los clientes que atienden las empresas seleccionadas y que ofrecen servicios en la GAM.

2. Establecer los costos percibidos como relevantes por parte de las empresas consultadas que ofrecen servicios en la GAM.

3. Definir los elementos considerados por los empresarios consultas en la comodidad al ofertar los servicios.

4. Proponer la estrategia de comunicación integrada de mercadeo para las empresas que ofrecen servicios al mercado nacional.

El tipo de estudio utilizado es cualitativo, en el que se emplean categorías de análisis, lo que permite identificar calidades esperadas del servicio (Taylor y Bogdan, 1987).

Con base en los objetivos formulados, las categorías de análisis empleadas en este estudio son perfil de los clientes, costos relevantes, comodidad del servicio y comunicación integrada de mercadeo, las cuales tienen sustento en el desarrollo teórico.

Se realizan entrevistas a quince líderes de organizaciones de servicios ubicadas en la GAM. Dichas entrevistas están conformadas por diecinueve preguntas abiertas.

De acuerdo con Barrantes (2013), la entrevista es "una técnica de recolección de datos que presta un importante servicio a la investigación cualitativa" (pág. 15).

La finalidad de la investigación en este caso es aplicada, porque busca crear la mejor solución o tratamiento mercadológico al mercadear servicios, a la luz de las ciencias administrativas y contables.

En forma similar, Hernández Sampieri, Fernández Collado y Baptista Lucio (2010) manifiestan que "en la indagación cualitativa, los investigadores deben construir formas inclusivas para descubrir las visiones múltiples de los participantes y adoptar papeles más personales e interactivos con ellos" (pág. 410).

El alcance de este estudio es temporal, es decir, transversal, porque recopila información de los líderes responsables del área de mercadeo en las organizaciones que ofrecen servicios en la Gran Área Metropolitana durante un momento determinado (Barrantes, 2013, pág.64).

Las fuentes primarias de información son quince profesionales, quienes toman las decisiones estratégicas en cuanto al mercadeo que se desarrolla en sus representadas. Estos informantes se seleccionan en forma intencional, por conveniencia; método conocido también como selección abierta, dentro de la estrategia cualitativa, es decir, no genera representatividad socioestructural de la población, por lo que no se generalizan los resultados al resto de la población. Se consideran en la muestra los casos políticamente importantes, debido a que cumplen con dos características filtro: trabajar en organizaciones que ofrecen servicios al mercado de la GAM y que sean tomadores de decisiones en ellas.

Según Barrantes (2013), en los estudios cualitativos "los pasos por seguir en estas investigaciones varían de un experto a otro; en general, pueden distinguirse las siguientes etapas: fase preparatoria, trabajo de campo, fase analítica y fase informativa" (pág. 146). 


\section{HALLAZGOS}

\section{Estrategias de mercadeo empleadas por las organizaciones que ofertan servicios.}

Los informantes se caracterizan por poseer altos niveles de escolaridad, ya que la mayoría cuentan con posgrados, principalmente en el área de administración de negocios, con distintos énfasis. En todos los casos, los informantes cuentan con más de cinco años de trabajar en la organización y ocupan puestos de dirección dentro de las organizaciones que representan. Algunos de ellos son los propietarios del negocio, cuyas actividades empresariales son asesoría en seguros, consultoría legal, tecnologías de la información (producción de software), mantenimiento en computadoras (hardware), municipalidad, educación, comercial, turismo, restaurante e ingeniería civil (consultoría).

Los mercados a los cuales estas organizaciones dirigen su oferta de servicios son los siguientes:

Mercado de consumidores, tales como familias, como unidad básica de consumo; estudiantes, ya sea de secundaria o de universidades; y personas físicas en general. Además, usuarios de los servicios de telecomunicaciones y el servicio de entretenimiento, ejecutivos y dueños de pequeñas y medianas empresas y organizaciones.

Dentro del mercado organizacional o de negocios, se dirigen a empresas transnacionales, corporativas, intermediación de seguros, servicios públicos, empresas pequeñas, medianas y corporaciones.

Los clientes actuales y potenciales de los entrevistados están ubicados en todo el territorio nacional y en algunos casos fuera de las fronteras costarricenses.

Varios de los informantes manifiestan atender además a públicos que pertenecen al mercado de consumidores, es decir, personas físicas que compran poco volumen de servicios.

La comodidad es un factor importante en la selección de la empresa proveedora, ya que la mayoría de los informantes manifiestan que elementos como cercanía, infraestructura adecuada, oferta directa del servicio, trato personalizado, conocimiento de procesos, garantía y servicio posventa, son esenciales en la percepción de la calidad percibida.

En cuanto al uso de la publicidad como herramienta de comunicación utilizada por las empresas de servicios que representan los informantes, entendida como el uso de medios masivos en donde existe un patrocinador identificado responsable del pago de la pauta comercial, la mayoría señala que no hacen uso de ella en forma masiva, sino que emplean estrategias de mercadeo directo y, en uno de los casos, utilizan revistas dirigidas a un segmento en particular. Asimismo, emplean herramientas publicitarias como vallas, patrocinios en actividades desarrolladas por la cámara de intermediarios de seguros y la asociación de supervisores de seguros latinoamericana, material en el punto de venta, redes sociales e Internet. Adicionalmente, emplean el uso de referidos, que contribuyen a la construcción de bases de datos y al "boca a boca".

Solamente uno de los informantes manifiesta que sí emplea medios publicitarios, con la contratación de agencias de publicidad, la cual coordina con el departamento de mercadeo dedicado a este tema, haciendo uso, además, de diferentes canales, ya sean escritos, pautas en radio y televisión, y redes sociales, entre otros.

Cabe destacar que muchos de ellos no emplean publicidad, debido a que los mercados objetivos a los cuales se dirigen se caracterizan por ser organizacionales, es decir, pocas personas físicas o jurídicas que adquieren gran volumen de producto.

Una de las herramientas de la estrategia de comunicación integrada de mercadeo más utilizada por estas empresas, según los informantes, es relaciones públicas, la cual ejecutan por medio de contactos de alto nivel. Asimismo, emplean referencias directas y sitio web, así como Facebook. El personal de ventas y servicio al cliente está en contacto directo con los clientes. Utilizan además herramientas como Waze, Google Ads Words y Tripadvisor, principalmente.

Otros informantes manifiestan que, de momento, no se han realizado campañas de relaciones públicas por parte de su representada. Lo que sí han implementado es el volanteo, para informar a los clientes de los servicios ofrecidos. Es 
interesante observar que enmarcan el volanteo dentro de la estrategia de RR. PP., cuando realmente está asociada con publicidad. En esta misma línea, también se emplean las redes sociales.

Ninguno de los informantes manifiesta que en exista un departamento de relaciones públicas o un protocolo de atención formalmente establecido para ello. Suelen confundir este elemento de la mezcla promocional con otras herramientas propias de publicidad, de promoción de ventas y de mercadeo directo.

En cuanto a la implementación de la estrategia de promoción de ventas para comunicarse con sus clientes, varios de ellos señalan que no la emplean. Por lo general, realizan promociones por medio de redes sociales y en artículos de boletines; el área de Mercadeo y Ventas es la encargada de gestionar las promociones que se llevan a cabo en las diferentes temporadas (ejemplo, Día de la Madre, fiestas patrias y partidos de la Selección Nacional de Fútbol, entre otros), en la mayor parte de estas organizaciones. En el caso de las relaciones públicas, en caso de emplearlas, se hace con eventos y actividades dirigidas.

Al consultar si emplean ventas personales para comunicarse con sus clientes, la mayoría señala que sí y, en general, es el principal medio de comunicación con los clientes. Dos informantes manifiestan que no utilizan la venta personal, por tratarse de ser servicios básicamente de referencias y por ser una entidad intermediaria de seguros.

En el caso de entidad educativa, señala que sí trabajan con venta personal, "por medio de empresas y prospección, que son visitas en los colegios". Sin embargo, es necesario considerar que la venta personal se da también en la ventanilla de servicios del punto de venta, lo cual ofrece una oportunidad para realizar el proceso adecuado, manejar objeciones y realizar el cierre y seguimiento, en forma oportuna y efectiva. Así se logra deleitar al cliente no solamente fuera de las instalaciones de la empresa, sino en las mismas, al contar con elementos como un servicio de excelencia y así garantizar recompras a futuro del mismo cliente o de sus recomendados.

En cuanto al uso de medios electrónicos para comunicarse con sus clientes, todos los informantes señalan que sí los emplean, mediante las vías existentes en Internet, en el campo virtual, plataformas digitales, sitio web, Facebook y otras redes sociales. En el caso de servicios municipales, se emplean para el pago de los servicios; existe una alianza con varias plataformas digitales de los bancos para que los pagos se puedan realizar en línea.

Para las empresas de tecnología de información, el precio de los servicios ofrecidos está en función de la calidad del servicio y promover una excelente "experiencia al cliente". En el caso de las municipalidades, el precio de los servicios está regulado por lo que prescribe el Código Municipal en su artículo 74 y la ley permite un $10 \%$ de utilidad para desarrollar el servicio.

En general, los informantes señalan que, a criterio de ellos, el costo más relevante es el salario y sus accesorios; para otros, las comisiones. Además, manifiestan que, cuando han acudido a financiamiento, el costo por concepto de intereses les resulta muy oneroso.

En el campo de las empresas de consultoría, el precio por hora profesional está regulado por el colegio profesional al que pertenecen. En tanto que, para las empresas de telecomunicaciones consultadas, la competencia existente, así como la oferta y demanda de los servicios, influyen directamente en el precio de los servicios ofrecidos.

Los representantes de las organizaciones consultados, mencionan que han enfrentado los cambios organizacionales a través de la incorporación de elementos como la comunicación vía digital, uso de nuevas tecnologías, software, contrataciones de personal de mando, capacitación intensiva al personal en materia inherente al servicio que brindan, servicio al cliente y automatización de gestiones manuales, así como también mediante alianzas estratégicas e innovación. Otras organizaciones lo han logrado adaptándose a las necesidades de los usuarios, siguiendo los pasos de la competencia y el comportamiento del mercado, mejoras en el entorno físico, atención personalizada y calidad. Otras empresas mencionan que agilizando el proceso de toma de decisiones y de adaptación a los requerimientos del mercado. 
Los informantes pertenecientes a las empresas comerciantes de seguros mencionan que el conocimiento y experiencia en el mercado de los seguros en Costa Rica les ha permitido alcanzar ventaja competitiva.

En general, los informantes mencionan que el precio psicológico y el nivel del mercado y la demanda, siguen siendo factores importantes a considerar, ya que factores como el posicionamiento respaldan la calidad de servicio percibida por los usuarios de este.

Como resultado general, es posible afirmar que las personas que dirigen las organizaciones en la GAM no son plenamente conscientes de los esfuerzos asociados en forma sistemática con procesos de comunicación integrada de mercadeo y específicamente de las diferencias sutiles que tiene la administración y gestión de mercadeo de servicios, que comúnmente se asocia con estrategias de $4 \mathrm{C}$.

\section{CONCLUSIONES}

Con base en los objetivos planteados, es posible concluir que las organizaciones de servicios consultadas en la GAM en su mayoría, están siendo lideradas por profesionales con altos niveles de escolaridad, cuyos puestos de trabajo están asociadas a áreas gerenciales, es decir, que son líderes de opinión y tomadores de decisiones. Sin embargo, el área de formación profesional en la mayoría de ellos no está asociada directamente con el mercadeo, por lo que desconocen los elementos comunes a la mezcla de mercadeo de servicios, mezcla promocional y estrategia de $4 \mathrm{C}$.

El perfil de clientes a los que sirven las organizaciones consultadas es muy variado y pertenecen tanto al mercado de consumidores, como al organizacional o de negocios.

En cuanto a los costos percibidos como relevantes, se observa la nómina (salarios, cargas sociales y todos los extremos laborales) y el costo financiero (intereses) cuando es necesario el acceso a recursos ante las entidades financieras para costear las operaciones normales y emprender nuevas actividades de negocio.

Los elementos percibidos como cómodos por los informantes son la ubicación, el personal capacitado, la infraestructura, las garantías y por supuesto las políticas de servicio al cliente. Estos son fundamentales en la prestación del servicio y en la disminución de las brechas en la oferta.

Las estrategias de comunicación integrada de mercadeo que emplean las organizaciones de servicios son principalmente mercadeo digital, venta personal y relaciones públicas. Muy pocas empresas de servicios implementan estrategias de promoción de ventas y publicidad masiva por medio de canales convencionales de comunicación como la televisión y no diferencian las características particulares de la administración de servicios.

Muchos de ellos no emplean publicidad, debido a que el mercado al cual se dirigen es organizacional, en donde existen pocas personas físicas o jurídicas que adquieren gran volumen de producto.

A través de esta investigación, se concluye que las organizaciones entrevistadas no están familiarizadas o identificadas con un sistema de costeo para el sector servicios, por lo que se recomienda el sistema de costeo por actividades (ABC), dado que este método permite la identificación, asignación, control y registro del costo de un recurso que genera cada actividad que agrega valor, considerando el uso del tiempo como inductor para la asignación a la hora de definir su precio.

Se concluye entonces que las organizaciones a las cuales se ha realizado la consulta se caracterizan porque comercializan servicios en la Gran Área Metropolitana costarricense y no suelen emplear todas las herramientas de la estrategia promocional, ni la de 4 C. Estas empresas las utilizan de acuerdo con su propio criterio, sin necesariamente conocer las estrategias recomendadas a nivel de mercadeo de servicios.

Por lo anterior, se recomienda que este tipo de organizaciones implementen la estrategia de mercadeo de servicios propuesta por Marcos Cobra (2000), con el fin de garantizar el éxito comercial y, por lo tanto, el logro de los objetivos. 


\section{REFERENCIAS}

Barrantes Echavarría, R. (2013). Investigación: un camino al conocimiento. San José: EUNED.

Beverinotti, J.; Chang, J.; Corrales, L. F. y Vargas, T. (2014). Diagnóstico de crecimiento para Costa Rica. Costa Rica: Banco Interamericano de Desarrrollo.

Cobra, M. (2000). Marketing de servicios. Colombia: McGraw Hill.

Córdoba López, J. (2009). Del marketing tradicional al marketing radical. Entramado, vol. 5, núm. 1, enero-junio, 2009, pp. 6-17 Universidad Libre Cali, Colombia.

Hill, S. (2000). Radical marketing. New York: HarperCollins.

Hoffman, D. (2002). Fundamentos de marketing de servicios. México: Tomson.

Hoffman, D. y Bateson, J. (2012). Fundamentos de marketing de servicios. México: Cengage Learning.

Govindarajan, V. y Shank, J. K. (1998). Gerencia estratégica de costos. Colombia: Norma.

Kotler, P. (2017). Marketing for competitiveness. Singapure: World SCientific Publishing Corp.

Kotler, P.; Bloom, P. y Hayes, T. (2016). El marketing de servicios profesionales. España: Paidós.

Hernández Sampieri, R.; Fernández Collado, C. y Baptista Lucio, P. (2010). Metodología de la investigación. México: McGraw-Hill.

Lovelock, C. (2016). Services marketing. London: World Scientific.

Mallo, C.; Gago, M. y Meljem, S. (2000). Contabilidad de costos y estratégica de gestión. España: Prentice Hall.

Morillo, M. (2005). Análisis de la cadena de valor industrial y de la cadena de valor agregado para las pequeñas y medianas industrias. Venezuela: Actualidad Contable FACES, 8(10).

Rivera Camino, J. y de Juan Vigaray, M. D. (2002). La promoción de ventas: variable clave del marketing. Madrid: ESIC.

Taylor, S. J. y Bogdan, R. (1987). Introducción a los métodos cualitativos de investigación: la búsqueda de significados. España: Paidos.

Tofler, A. (1997). La creación de una nueva civilización. España: Plaza \& Janes.

Treviño, R. (2010). Publicidad. México: McGraw-Hill Interamericana.

Wilcox, D. (2012). Relaciones públicas. México: Prentice-Hall. 